\title{
Evolution, Kultur und Natur
}

\author{
Korrespondenzadresse \\ Prof. Dr. Dr. Manfred Spitzer \\ Universität Ulm \\ Leimgrubenweg 12-14 \\ 87054 Ulm, Deutschland
}

\author{
Bibliografie \\ Nervenheilkunde 2021; 40: 400-405 \\ DOI 10.1055/a-1389-6521 \\ ISSN 0722-1541 \\ (C) 2021. Thieme. All rights reserved. \\ Georg Thieme Verlag KG, Rüdigerstraße 14, \\ 70469 Stuttgart, Germany
}

Bis vor gut einem halben Jahrhundert waren Natur und Kultur fein säuberlich sortiert: Für das eine waren die Naturwissenschaften zuständig und für das andere die Geistes- oder Kulturwissenschaften. Zwischen beiden gab es eine „friedliche Koexistenz“, solange es nicht ums Geld ging. Der wissenschaftliche Alltag war von wechselseitiger Ignoranz und Geringschätzung geprägt. Lediglich in Sonntagsreden wurde zuweilen die lange gemeinsame Geschichte betont. Wenn es dann doch gelegentlich ums Geld ging, dann hatten die Geisteswissenschaften immer schon verloren - fast im philosophischen, prinzipiell gemeinten Sinn des „immer schon“. Ein neues Gebäude für die Molekularbiologie oder ein ganzer neuer Campus für die Naturwissenschaften? - „Kein Problem“. Renovierung irgendeines uralten Gebäudes für die Sprachwissenschaften? - „Kein Geld“. Dabei gehörten die in den 1970er-Jahren zunächst sehr zaghaft aufkommenden Berührungen zwischen den Geistes- und Naturwissenschaften zum Spannendsten, was ich während meiner Studienzeit erlebt habe. Wir Menschen gehören nun einmal auch zur Natur und unsere Kultur damit ebenfalls. Diese ist hierdurch jedoch keineswegs vollständig determiniert, sondern kann vielmehr auch unabhängig von Natur eine Eigendynamik entwickeln, die anderen Gesetzen folgt als den Naturgesetzen. Entsprechend haben Studien gefunden, dass geschichtliche Zufälle Ursache kultureller Unterschiede sein können [14, 15]. Allein die Natur reicht nicht aus, um die historisch gewachsenen Unterschiede zwischen verschiedenen Kulturen zu erklären.

Dennoch ist gerade das komplexe Wechselspiel von Natur und Kultur interessant, vor allem, wenn es um die Einordnung dessen, was ist, was sein soll und überhaupt sein kann und unsere Existenz (Ontologie, Ethik, Metaphysik) und unser Erkenntnisvermögen (Erkenntnistheorie) geht. Anhand der Beispiele von Farben [24] und Emotionen [11] konnte gezeigt werden, dass man globale Unterschiede in der sprachlichen Sortierung der Phänomene weder allein mit Sprache und Kultur noch allein mit Physiologie und Physik erklären kann. Anhand zweier Studien sei in der Folge dargelegt, dass das Zusammenspiel von Natur und Kultur nie spannender diskutiert wurde als in der Gegenwart.

\section{Natur als Motor kultureller Unterschiede}

Eine im Fachblatt Science publizierte Studie mit dem simplen Titel „Lokale Konvergenz des Verhaltens über Arten hinweg“ ging der Frage nach, wie sich Lebensbedingungen bzw. Umweltfaktoren auf das Verhalten von Menschen auswirken [2]. Untersucht wurden Verhaltensweisen in Verbindung mit Nahrungssuche, Paarung und sozialer Interaktion. Hierzu wurde eine große ethnografische Datenbank mit Beobachtungen vor allem aus dem vorletzten Jahrhundert zum Verhalten von 339 Stämmen von Jägern und Sammlern aus der ganzen Welt herangezogen: 20 Stämme aus Afrika, 28 aus Asien, 56 aus Australien, 215 aus Nordamerika und 20 aus Südamerika. Man kann bei relativ kleinen, räumlich begrenzt lokalisierten Stämmen davon ausgehen, dass sie von dem lebten, was die Natur in ihrer Umgebung bot und kaum Handel trieben. Und so fanden sich tatsächlich klare Auswirkungen bestimmter Aspekte dieser Natur auf die 3 Gruppen von Verhaltensweisen. Sind beispielsweise die Nahrungsquellen unzuverlässig, sollten mehr Verhaltensweisen zu beobachten sein, die sich auf das Speichern oder Konservieren von Nahrung beziehen. Neben dem Aufbewahren von Nahrungsmitteln wurden auch die Größe des Bereichs, in dem Nahrungsmittel gesucht werden, sowie das Wanderungsverhalten zur Nahrungssuche erfasst. Im Hinblick auf das Paarungsverhalten wurde das Alter bei der ersten Elternschaft, das Praktizieren von Monogamie oder Polygynie, die Regeln beim Heiraten (Endogamie, d. h. bei Eheschließungen entstammt der Partner bevorzugt oder vorgeschriebener Weise der eigenen sozialen Gruppe, Großfamilie, Stammesgruppe oder sozialen Schicht, oder nicht, d. h. Exogamie) und die Scheidungsrate festgehalten. Beim Sozialverhalten wurde das Ausmaß des väterlichen Anteils am Großziehen der Kinder, die Populationsdichte, die Gruppengröße und die Bildung von gesellschaftlichen Klassen (oder deren Abwesenheit) untersucht. All diese Variablen könnten durch Unterschiede in den natürlichen Umweltbedingungen wie Nahrungsmittelverfügbarkeit (Pflanzenwuchs, Tiere), Vorhandensein von Raubtieren, Jahreszeiten etc. beeinflusst werden, aber auch durch kulturelle Unterschiede bedingt sein.

Der Witz der Studie bestand nun darin, dass man diese Daten zum Verhalten von Menschen mit entsprechenden Daten zum Verhalten von anderen Säugetieren ${ }^{1}$ und Vögeln verglich. Hierzu wurden aus weiteren Datenbanken Säugetiere und Vögel identifiziert, deren lokale Verbreitung mit den Gebieten der Stämme

1 Im Folgenden sind grundsätzlich diese „anderen“, also nicht menschlichen, Säugetiere gemeint, wenn von „Säugetieren“ die Rede ist. 
überlappte. Auch bei Tieren lassen sich die Größe des Bereichs zur Suche von Futter, die Gruppengröße oder die Bildung sozialer Klassen untersuchen. Migration wurde nur bei (Zug-)Vögeln untersucht, die Größe des Bereichs der täglichen Nahrungssuche nur bei Säugetieren. Um den Einfluss von Natur und Kultur auf das menschliche Verhalten zu untersuchen, bestimmte man das Ausmaß des Zusammenhangs zwischen menschlichem und tierischem Verhalten. Unter der Annahme, dass tierisches Verhalten keiner „Kulturgeschichte“ unterliegt ${ }^{2}$, sondern durch die Natur bzw. Aspekte des Lebensraums bestimmt wird, lässt sich am Ausmaß des Zusammenhangs zwischen menschlichem und tierischem Verhalten abschätzen, wie weit das menschliche Verhalten ebenfalls durch die Natur bedingt ist - und nicht das Ergebnis historisch gewachsener Zufälle darstellt, die man zusammenfassend „Kultur“ nennt.

Wie aber misst man die Scheidungsrate, die Bildung sozialer Klassen oder der Anteil des Vaters an der Erziehung der Nachkommen bei Tieren? Um dies zu erfahren, muss man tief in die 82 Seiten des digitalen Supplements zur dreieinhalbseitigen auf Papier gedruckten Arbeit einsteigen. Nur bei Vögeln ließ sich die Scheidungsrate abschätzen, ohne genetische Proben zu untersuchen, was bislang noch nicht in dem Ausmaß geschehen ist, wie es für die vorliegende Arbeit nötig gewesen wäre. Man verwendete hierzu Daten zum jährlichen Partnerwechsel (ja/nein), die für viele Vogelarten publiziert sind. Bei Vögeln besteht zudem ein mehr oder weniger stark ausgeprägter Unterschied des Federkleids zwischen Männchen und Weibchen. Bei manchen Vogelarten kann man beide fast nicht unterscheiden, wohingegen bei anderen Arten das Männchen wesentlich stärker und bunter gefiedert ist als das Weibchen. Dieser Unterschied zwischen den Geschlechtern geht mit männlichem Balzverhalten und Polygynie einher, wie man seit langem weiß. Sehen das Männchen und das Weibchen hingegen (nahezu) gleich aus, verhalten sich die Tiere eher lebenszeitlich monogam.

Was die soziale Klassenzugehörigkeit anbelangt, so ging es beim Menschen vor allem um „das Ausmaß, in dem Individuen innerhalb einer Gemeinschaft unterschiedliche Rollen und Fortpflanzungserfolge haben könnten. Bei als Jäger und Sammler lebenden Menschen stellt die Rolle eines Anführers eine Form der sozialen Klassenunterscheidung dar. Personen, die Anführer sind, haben meist einen höheren Fortpflanzungserfolg “3, schreiben die Autoren [2]. Dann fahren sie mit Säugetieren und Vögeln wie folgt fort: „Säugetiere leben oft in kooperativen Gesellschaften, in denen es ein dominantes Zuchtpaar gibt, das die meisten Nachkommen hervorbringt, und daneben erwachsene untergeordnete nicht direkt zur Reproduktion beitragende Helfer, die sich um die Nachkommen des dominanten Paares kümmern. Individuen unterscheiden sich dementsprechend sowohl in ihrer Rolle als auch in ihrem Fortpflanzungserfolg. Vögel leben ebenfalls oft in

2 Diese Annahme galt noch vor wenigen Jahrzehnten als selbstverständlich. Mittlerweile wurde sie problematisch, denn auch bei Tieren kommt Kultur vor, d. h. tradiertes, gelerntes Verhalten [21].

3 „[...] the extent to which individuals within a society might have different roles and reproductive success. For humans, institutionalized leadership in hunter-gatherer populations represents a form of social class distinction. Individuals who are leaders generally tend to have higher reproductive success."
Gesellschaften nicht verwandter Tiere, in denen es ein einziges dominantes Brutpaar und weitere erwachsene Tiere gibt, die sich der Gruppe angeschlossen haben, sich aber nicht vermehren. Je nach eingenommener Rolle gibt es einen Unterschied im reproduktiven Erfolg."4

Der folgende Satz zeigt, wie detailreich und methodisch differenziert die Überlegungen der Autoren zu den einzelnen untersuchten Variablen sind: „Wir haben keine kooperativen Züchter von Verwandten [in die Gruppe der untersuchten Vögel] eingeschlossen, da diese im Allgemeinen Arten widerspiegeln, bei denen nicht zerstreute Individuen die Nachkommen eines dominanten Paares versorgen. Diese nicht zerstreuten Individuen sind möglicherweise noch keine erwachsenen Tiere, daher spiegelt sich [in diesen Gruppen] die Schichtung nach Alter und nicht nach Rolle [bzw. Stand] wider. "5 Die Ergebnisse der Studie sind bemerkenswert, denn für die meisten der untersuchten Variablen zeigten sich ähnliche Effekte bei Mensch und Tier. „Wir beobachteten Zusammenhänge zwischen der Abhängigkeit des Menschen von Nahrungsvorräten und dem Anteil der lokalen Säugetiere und Vögel, die Nahrung horten. Auch gab es Assoziationen bei den kurzfristigen Ausflügen zur Beschaffung von Ressourcen, wobei menschliche Populationen an Orten, an denen Säugetiere längere Tagesstrecken zur Nahrungssuche zurücklegen, ebenfalls zentral lebende Futtersammler sind (was in der Regel mit längeren Tagesstrecken aufgrund lokaler Ressourcenverknappung verbunden ist. Zu Vögeln sind keine Daten verfügbar)“, ${ }^{6}$ schreiben die Autoren hierzu [2].

Ähnlich war es auch beim Reproduktionsverhalten: „Wir fanden die folgenden Zusammenhänge im Verhalten: Globale Variationen im Alter der ersten Fortpflanzung sind bei Menschen, Säugetieren und Vögeln miteinander korreliert. An bestimmten Orten ist es wahrscheinlicher, dass Männchen die Paarungen monopolisieren; wo ein höherer Anteil der menschlichen Männer mit mehreren Frauen verheiratet ist, leben auch mehr Säugetiere in instabilen Gruppen (was ein Monopolisierungspotenzial bietet) und investieren männliche Vögel mehr in ihr Gefieder, um mehrere Weibchen anzuziehen. [...] Trennungen zwischen Säugetier-Paarungspartnern sind in denjenigen Gebieten wahrscheinlicher, wo Scheidungen in menschlichen Populationen erlaubt sind und Vogelpaare mit höhe-

4 „For mammals, cooperative breeders are societies in which there is a dominant breeding pair who produce most of the offspring and adult subordinate non-reproducing helpers who care for the offspring of the dominant pair. Individuals accordingly differ both in their role and in their reproductive success. For birds, non-kin cooperative breeders are societies in which there is generally a single dominant breeding pair and adults who have joined the group but do not reproduce. Again, there is a distinction between individuals in roles and reproductive success."

5 „We did not include kin cooperative breeders in birds because these generally reflect species in which non-dispersed individuals provide care to the offspring of a dominant pair. These non-dispersed individuals might not yet be adults, so the stratification is reflected by age and not by role."

6 „We observed associations between the reliance of humans on food storage and the proportion of local mammals and birds that hoard food. There were associations in short-term movements to acquire resources, with human populations being central-place foragers (which is usually associated with longer day ranges because of local resource depletion) in locations where mammals have longer daily foraging trips (no bird data available).“ 
rer Wahrscheinlichkeit sich jedes Jahr trennen “7 [2]. Sogar im Hinblick auf das allgemeine Sozialverhalten und die Erziehung scheinen sich die Menschen mit den anderen Säugetieren und Vögeln einig zu sein ${ }^{8}$ : „[...] an Orten, an denen Männer einen höheren Anteil der Nahrung für ihre Familie bereitstellen, tragen Männchen bei einem höheren Anteil von Säugetierarten zur Fütterung und zum Tragen des Nachwuchses bei und sind bei einem höheren Anteil von Vogelarten die alleinigen Anbieter der elterlichen Betreuung. Wo Menschen in höheren Dichten leben, tun dies auch andere Säugetiere und Vögel. An Orten, an denen die Wohngruppengröße von Menschen größer ist, sind die sozialen Gruppengrößen von Säugetieren größer und Vögel gehen eher in Gruppen auf Nahrungssuche, als dass sie sich auf die einsame Nahrungssuche verlassen. Wo menschliche Populationen soziale Klassen haben, haben mehr Säugetiere und Vögel ein soziales System mit dominanten Brütern und untergeordneten, nicht brütenden Helfern “ [2].

Diese Daten verdeutlichen, dass Umweltbedingungen das Verhalten von Menschen und Tieren auf ähnliche Weise beeinflussen. Möglicherweise sind die Ähnlichkeiten im Verhalten von Menschen, Säugetieren und Vögeln auf den Selektionsdruck der lokalen Umgebung zurückzuführen. Dafür spricht, dass die Assoziationen zwischen den Arten geringer werden, wenn ökologische Variablen als Kovariablen (z. B. Breitengrad, Höhe, Küstennähe und Biome, also Gemeinschaften von Pflanzen- und Tierarten) zur Varianzerklärung des Verhaltens in die Analyse einbezogen werden. Anders gesagt: Rechnet man die Ökologie heraus, werden die Effekte kleiner. Folglich muss es auch an der Ökologie liegen. Ebenfalls für diesen Zusammenhang von Ökologie und Verhalten spricht, dass die Assoziationen zwischen denselben ökologischen Variablen und Verhaltensweisen bei Menschen, Säugetieren und Vögeln sehr ähnlich sind. Schließlich braucht man gar nicht Daten von gleichen Orten zu untersuchen, sondern findet entsprechende Zusammenhänge auch bei der Untersuchung von ökologisch ähnlichen Orten, die geografisch weit voneinander entfernt liegen. Dies legt einen konsistenten Einfluss ökologischer Faktoren nahe.

Dennoch sind diese Ergebnisse nicht einfach zu interpretieren, denn es wurden verschiedene Arten von Säugetieren und Vögeln untersucht, also bei den Tieren die Varianz des Verhaltens zwischen

7 „We found the following connections in behavior: (i) Global variation in the age of first reproduction is linked across humans, mammals, and birds. (ii) Males are more likely to monopolize matings in certain places, with a higher proportion of human men being married to multiple women, more mammals living in unstable groups (providing monopolization potential), and male birds investing more into their plumage to attract multiple females. [...] (iv) Splits between mating partners are more likely in some areas, with divorce permitted in human populations and bird pairs more likely to split up each year."

8 Ich zitiere hier die Autoren nochmals im Original, weil manch ein Leser sonst möglicherweise glaubt, ich würde übertreiben: „(i) [...] in locations in which human males provide a higher proportion of the diet for their family, males contribute to the feeding and carrying of offspring in a higher proportion of mammal species and are the sole providers of parental care in a higher proportion of bird species. (ii) Where humans live in higher densities, so do other mammals and birds. (iii) In locations in which residential group sizes in humans are larger, social group sizes of mammals are larger and birds are more likely to forage in groups than to rely on solitary foraging. (iv) Where human populations have social classes, more mammals and birds have a social system with dominant breeders and subordinate nonbreeding helpers."
Arten, beim Menschen hingegen wurde die Varianz des Verhaltens innerhalb einer Art bestimmt. Die unterschiedlichen Verhaltensweisen der Arten sind evolutionär entstanden und werden genetisch weitergegeben, die unterschiedlichen Verhaltensweisen des Menschen hingegen sind kulturell entstanden und werden kulturell tradiert. Interessant ist dennoch, dass biologische Evolution und kulturelle Innovation aufgrund ökologischer Gegebenheiten ganz offensichtlich konvergent verlaufen können, was bedeutet, dass Kultur weniger „zufällig“ oder gar „beliebig“ entsteht als zuvor gedacht. Sie folgt vielmehr - wie die Evolution - zumindest teilweise aus den Umweltbedingungen. Dies wirft die Frage auf, ob die Verhaltensvielfalt moderner menschlicher Gesellschaften nach wie vor ökologische Bedingungen widerspiegelt, auch wenn Landwirtschaft, Marktintegration und Technologie die Reaktion des Verhaltens auf lokale Bedingungen modulieren können. Dies ist bislang nicht explizit untersucht. Es gibt jedoch Beispiele dafür, dass menschliche Kulturen tiefe Wurzeln haben und auch Jahrhunderte (Jahrtausende?) nach dem Wegfall von deren Entstehungsbedingungen noch Bestand haben $[18,20]$.

\section{Kultur als Motor der Evolution unserer Natur}

Wie eine Reihe entsprechender Beispiele zeigt, gibt es auch den umgekehrten Zusammenhang zwischen Natur und Kultur: Nicht die Natur treibt und verändert die Kultur, sondern die Kultur treibt und verändert unsere Natur. Dachte man früher, unsere Evolution habe seit Beginn unserer Kultur (und der geschichtlichen Zeit) aufgehört, denkt man heute ganz anders und spricht von einer kumulativen kulturell getriebenen Evolution des menschlichen Gehirns. Dies sei kurz anhand eines bekannten und in meinen Beiträgen zu dieser Zeitschrift bereits mehrfach erwähnten Beispiels der Unverträglichkeit von Milchzucker (Laktoseintoleranz) erläutert. Neugeborene Säugetiere bilden das Enzym Laktase, das Milchzucker (ein „Zweifachzucker“ aus der Verbindung von Glukose und Galaktose), der vom Körper nicht aufgenommen werden kann, in die verwertbaren Einfachzucker Galaktose und Glukose spaltet. Laktase wird dazu in der Dünndarmschleimhaut gebildet, befindet sich im Saft des Dünndarms und ermöglicht dem Neugeborenen die Verdauung der Muttermilch als zunächst einzige Energiequelle [10].

Im Laufe der natürlichen Entwöhnung von der Muttermilch sinkt bei allen Säugetieren die Laktaseaktivität auf etwa 5-10\% ab. Daher ist die Laktoseintoleranz, also der Verlust der Fähigkeit, Milchzucker zu verdauen, für alle ausgewachsenen Säugetiere und damit auch für uns Menschen der Normalfall. Warum sollte der Körper eines Säugetiers auch zeitlebens ein Enzym - die Laktase - produzieren, das nach der Säuge- bzw. Stillperiode nicht mehr gebraucht wird? Nur bei Völkern, die seit Jahrtausenden Viehzucht und Milchwirtschaft betreiben, sich also auch als Erwachsene noch von Milch ernähren, hat sich eine Mutation durchgesetzt, die dazu führt, dass die Produktion von Laktase nicht abgeschaltet wird, sodass auch noch im Erwachsenenalter genügend Laktase im Dünndarm vorhanden ist; dies wird als Laktasepersistenz bezeichnet. Diese Mutation war von großem Vorteil, konnten doch die Menschen erst mit ihr den Vorteil der Milchwirtschaft (einschließlich Käseherstellung und damit Haltbarmachung von lebenswichtigem Eiweiß) für ihre Ernährung richtig nutzen. Es kam sogar zu einer kulturell (Milchwirtschaft) getriebenen Koevolution von Genen für die 
Milchproduktion bei Kühen und Genen zur Milchverdauung beim Menschen [3]. Die Mutation setzte sich damit durch, je früher sie auftrat, desto mehr: In Gegenden, wo es Viehzucht schon seit Jahrtausenden gibt, ist die Laktoseintoleranz viel seltener als in Gegenden, wo erst seit kürzerer Zeit Milch und Milchprodukte zum Speiseplan gehören. In Japan beispielsweise ist das erst seit einigen Jahrzehnten der Fall, weswegen der Anteil der Bevölkerung mit Laktoseintoleranz sehr hoch ist. Die Datenbasis für diese Sicht der Dinge hat sich im vergangenen Jahrzehnt dank paleogenetischer Studien ${ }^{9}$ deutlich verbessert. Der Mainzer Anthropologe Joachim Burger publizierte im Jahr 2007 zusammen mit britischen Kollegen eine genetische Untersuchung von 97800 bis 7200 Jahre alten Skeletten aus 5 europäischen Fundstellen (Derenberg in Deutschland, Szarvas und Albertfalva in Ungarn, Kretuonas in Litauen). Laut DNA-Analyse konnte keiner dieser Menschen Milch verdauen; übrigens auch nicht der in Österreich gefundene etwa 5300 Jahre alte mumifizierte „Ötzi“ [12].

Eine Ende 2020 erneut von Burger publizierte genetische Studie fand auch an Skeletten von 14 Kriegern des Schlachtfeldes im Tollensetal (in Mecklenburg-Vorpommern, um das Jahr 1250 v. Chr.), an 18 Individuen aus der Bronzezeit-Grabungsstätte von Mokrin (in der serbischen Provinz Kikinda, etwa 2100 bis 1700 v. Chr.) und 37 Individuen aus der osteuropäischen Steppe nördlich des Schwarzen und Kaspischen Meeres (etwa 4000 bis 2000 v. Chr.) keine Laktasepersistenz, was den Autoren zufolge darauf hinweist, dass sich diese erst in den vergangenen 3000 Jahren entwickelt hat [5]. Dies wird gestützt durch ein etwa 1500 Jahre altes Skelett mit Laktosepersistenz aus Eltville in Frankreich, das als aktive Kontrolle in der Studie von Burger und Mitarbeitern diente, und durch eine im Jahr 2013 veröffentlichte genetische Analyse von 18 Skeletten eines mittelalterlichen Friedhofs im deutschen Dalheim, die bei 13 Skeletten (72\%) Laktasepersistenz nachwies [13]. Folgender Sachverhalt bestätigt diese Überlegungen nochmals eindrucksvoll: Normalerweise handelt es sich bei einem erblichen Defekt um eine ganz bestimmte Mutation. Der genetische „Normalfall“ - man spricht auch vom genetischen „Wildtyp“ - hingegen weist Unterschiede auf, d. h. beim Wildtyp handelt es sich um eine Vielzahl genetischer Varianten. ${ }^{10}$ Bei der Laktoseintoleranz ist dies umgekehrt, denn was heute „Krankheit“ ist (Laktose nicht verdauen zu können), war früher der Normalfall (und damit der variantenreiche Wildtyp), wohingegen der heutige Normalfall Ausdruck einer Mutation ist. In einer bestimmten geografischen Region liegt meist nur eine Mutation vor, deren Vorteil offensichtlich so groß war, dass sie sich in der Bevölkerung durchsetzte.

Fassen wir zusammen: Der Gedanke, die Evolution des Menschen hat nicht aufgehört, ist noch nicht besonders alt. Insbesondere der Gedanke, dass Kultur der Hauptreiber der menschlichen Evolution während der letzten hunderttausend Jahre war (plus/ minus ein paar Jahrzehntausende), ist erst nach der Jahrtausendwende wirklich salonfähig geworden [9, 23]. Zu unserer Kultur gehört auch Sprache, Schrift, sehr große Gesellschaften, Kriege

9 Es ist schon unglaublich, was man alles durch die Analyse von Genen aus Jahrtausende alten Funden herausgefunden hat [16].

10 In der Genetik ist der Wildtyp so definiert: Sein Genom ist natürlicherweise durch die Evolution entstanden. Genetische Abweichungen werden als Mutanten bezeichnet. Sie sind seltener als der Wildtyp. und seit einigen Jahrhunderten Naturwissenschaft und Technik. Die Zukunft wird zeigen, was wir noch alles an Analogien zur Laktoseintoleranz entdecken werden: „Schulunterrichtsintolerenz“ (Aufmerksamkeitsstörungen, die am Lagerfeuer in grauer Vorzeit zum Überleben der gesamten Gruppe beitrugen, wenn es irgendwo im Gebüsch geraschelt hat [25]), „hyperkalorische Nahrungsmittelintoleranz“ (Adipositas), Nationalismus und Fremdenfeindlichkeit (Hyperoxitocinismus [7, 8, 17]) und digitales Hyperablenkungssyndrom (Smartphone-Besitz). Wir können jetzt über dies alles viel klarer nachdenken.

\section{Literatur}

[1] Hill K, Boyd R. Behavioral convergence in humans and animals. Science 2021; 371: 235-236

[2] Barsbai T, Lukas D, Pondorfer A. Local convergence of behavior across species. Science 2021; 371: 292-295

[3] Beja-Pereira A, Luikart G, England P, et al. Gene-culture coevolution between cattle milk protein genes and human lactase genes. Nat Genet 2003; 35: 311-313

[4] Boyd R. A different kind of animal. How culture transformed our species. Oxford: Princeton University Press, 2018

[5] Burger J, Kirchner M, Bramanti B, et al. Absence of the lactase-persistence-associated allele in early Neolithic Europeans. PNAS 2020; 104: 3736-3741

[6] Burger J, Link V, Blöcher J, et al. Low Prevalence of Lactase Persistence in Bronze Age Europe Indicates Ongoing Strong Selection over the Last 3,000 Years. Current Biology 2020; 30: 4307-4315

[7] Choi JK, Bowles. The coevolution of parochial altruism and war. Science 2007; 318: 636-640

[8] De Dreu CKW, Greer LL, Handgraaf MJJ, et al. The neuropeptide oxytocin regulates parochial altruism in intergroup conflict among humans. Science 2010; 328: 1408-1411

[9] Henrich J. The Secret of our Success. Princeton: University Press, 2016

[10] Höffeler F. Geschichte und Evolution der Lactose(in)toleranz. Biologie in unserer Zeit 2009; 39: 378-387

[11] Jackson JC, Watts J, Henry TR, et al. Motion semantics show both cultural variation and universal structure. Science 2019; 366: 1517-1522

[12] Keller A, Graefen A, Ball M, et al. New insights into the Tyrolean Iceman's origin and phenotype as inferred by whole-genome sequencing. In: Nature Communications 2012; 3: 69

[13] Krüttli A, Bouwman A, Gülfirde A, et al. Ancient DNA Analysis Reveals High Frequency of European Lactase Persistence Allele (T-13910) in Medieval Central Europe. PLoS ONE 2014; 9: e86251

[14] Mathew S, Perreault C. Behavioural variation in 172 small-scale societies indicates that social learning is the main mode of human adaptation. Proc R Soc B 2015; 282: 20150061

[15] Mathew S, Perreault C. Cultural history, not ecological environment, is the main determinant of human behaviour. Proc R Soc B 2016; 283: 20160177

[16] Reich D. Who we are and how we got there. Oxford: University Press, 2018

[17] Samuni L, Preis A, Mundry R, et al. Oxytocin reactivity during intergroup conflict in wild chimpanzees. PNAS 2017; 268-273

[18] Schulz JF, Bahrami-Rad D, Beauchamp JP, et al. The Church, intensive kinship, and global psychological variation. Science 2019; 366: eaau5141 
[19] Smith JE, Gavrilets S, Mulder MB, et al. Leadership in mammalian societies: Emergence, distribution, power, and payoff. Trends Ecol Evol 2016; 31: 54-66

[20] Talhelm T, Zhang X, Oishi S, et al. Large-scale psychological differences within China explained by rice versus wheat agriculture. Science 2014; 344: 603-608

[21] Whiten A. The burgeoning reach of animal culture. Science 2021; 372: eabe6514
[22] Wilson EO. Sociobiology. Cambridge: Harvard University Press, 1975

[23] Wilson EO. The Social Conquest of Earth. New York: Norton, 2012

[24] Youn $\mathrm{H}$, Suttond L, Smith E, et al. On the universal structure of human lexical semantics. PNAS 2016; 113: 1766-1771

[25] Esteller-Cucala P, Maceda I, Børglum AD, et al. Genomic analysis of the natural history of attention-deficit/hyperactivity disorder using Neanderthal and ancient Homo sapiens samples. Scientific Reports 2020; 10: 8622 